\title{
Limited knowledge and practice of Chinese medical students regarding health-care associated infections
}

\author{
Yuanchun Huang ${ }^{1}$, Wenni $\mathrm{Xie}^{2}$, Jun Zeng ${ }^{3}$, Frieda Law ${ }^{4}$, William Ba-Thein ${ }^{3,5}$ \\ ${ }^{1}$ Department of Clinical Microbiology, First Affiliated Hospital of Shantou University Medical College, Shantou, \\ Guangdong, P.R. China \\ ${ }^{2}$ Office of Teaching Affairs, Shantou University Medical College, Shantou, Guangdong, P.R. China \\ ${ }^{3}$ Department of Microbiology and Immunology, Shantou University Medical College, Shantou, Guangdong, P.R. \\ China \\ ${ }^{4}$ Consultant Office, Shantou University Medical College, Shantou, Guangdong, P.R. China \\ ${ }^{5}$ Shantou-Oxford Clinical Research Unit, Shantou University Medical College, Shantou, Guangdong, P.R. China
}

\begin{abstract}
Introduction: Health-care associated infections (HCAIs) occur worldwide and affect both patients and health-care workers (HCWs), including medical students. This study aimed to investigate HCAI risks associated with clinical medical students attending Shantou University Medical College (SUMC) and the effectiveness of their learning resources.

Methodology: Four cohorts $(n=272)$ of medical students participated in a questionnaire-based survey was done on (year 5 in the 5 -year program and years 5 to 7 in the 7-year program) undergoing internship training in 14 teaching hospitals in Guangdong, China.

Results: The mean overall score of the students was $52.54 \pm 0.45$ (mean \pm SE). Students received fairly good scores in hand hygiene (77.57 \pm $0.77)$ and HCAI source $(63.16 \pm 1.18)$; relatively weak scores in the isolation precautions $(44.59 \pm 0.55)$, HCW safety (45.59 \pm 0.86$)$, and personal protective equipment (57.64 \pm 0.60$)$; and the weakest scores in HCA-pathogen identification (27.44 \pm 0.81$)$. The year of education $(\mathrm{r}=0.089, \mathrm{P}=0.144, \mathrm{n}=272)$ or internship placement $(\mathrm{r}=0.077, \mathrm{P}=0.206, \mathrm{n}=272)$ had no significant influence on their level of knowledge.

Conclusions: This study demonstrates that medical students at SUMC have limited knowledge and practice regarding HCAI due to substantial deficiencies in their learning resources. Review of medical curricula, improvement in preclinical and clinical training, and surveillance and monitoring of practicing HCWs are urgently needed to minimize risk of HCAIs in patients and HCWs.
\end{abstract}

Key words: medical student; healthcare-associated infection; infection control

J Infect Dev Ctries 2013; 7(2):144-151.

(Received 26 October 2012 - Accepted 14 January 2013)

Copyright $(\underset{2}{ } 2013$ Huanget al. This is an open-access article distributed under the Creative Commons Attribution License, which permits unrestricted use, distribution, and reproduction in any medium, provided the original work is properly cited.

\section{Introduction}

Health-care associated infection (HCAI) may cause prolonged hospital stays, higher mortality, longterm disability, increased microbial drug resistance, and excess health-care costs. The occurrence of HCAI at any given time is 15.5 per 100 patients in developing countries and 5 to 12 per 100 patients in developed countries. In Europe, there are more than 4.5 million episodes of HCAI, attributing to 37,000 deaths and costing $€ 7$ billion per year; in the United States, there were 1.7 million affected patients resulting in 99,000 deaths in 2002, and the estimated cost in 2004 was US\$ 6.5 billion [1]. In China, 400,000 annual occurrences of HCAI (with 8\% incidence) with more than RMB 1.5 billion in extra expenditures were reported by the Chinese Ministry of Health in 2010.

HCAIs can be transmitted between hospitalized patients and HCWs including health-care students. Recently HCAI has become a major issue in healthcare safety that concerns the safety of patients and HCWs, but it still has not received priority attention in the medical curriculum in many developing and some developed countries. Consequently, patients as well as HCWs, especially medical and nursing students undergoing clinical training, are at high risk of contracting HCAI due generally to lack of awareness and skills. For example, a study in southern Taiwan reported low level of knowledge concerning standard and additional precautions among pre-service nursing students. Their ability in applying these precautions in 
clinical practice also appeared to be inadequate [2]. Students' clinical skills and performance are greatly influenced by their learning resources, such as formal classroom lectures, bedside teaching during clinical rounds, hospital guidelines, and scientific articles. Although there are a number of reports concerning health-care students and HCAI in other countries, China has lagged behind in this aspect.

The objectives of this study were to monitor HCAI risks associated with clinical medical students and to explore the influence of students' learning resources on HCAI by evaluating their knowledge and practices regarding prevention and control of HCAIs using a comprehensively designed questionnaire in Shantou University Medical College (SUMC) and its affiliated hospitals.

\section{Methodology}

A cross-sectional, self-administered but supervised, anonymous, written questionnaire-based survey concerning HCAIs was conducted in SUMC in Guangdong province in southern China in May 2010.

The study was approved by the Ethics Committee of Shantou University Medical College.

\section{Background of study site and participants}

In SUMC where this study was conducted, students in the five-year program undergo one-year rotary internship training in all sub-specialties in their fifth year, after which they take the final exams. For the seven-year program, students do the same as those in the five-year program; however, their sixth and seventh years are spent in a sub-specialty area in the ward with clinical responsibility.

Participants in this survey were 272 senior medical students from SUMC undergoing clinical rotations (internship) in 14 medical college affiliated hospitals in Guangdong. Students were from four cohorts: year 5 students of the five-year program $(\mathrm{n}=51)$, and year $5(n=76)$, year $6(n=80)$, and year $7(n=65)$ of the seven-year program. The four cohorts will be hereafter referred to as $5 / 5,5 / 7,6 / 7$, and $7 / 7$, respectively.

\section{Questionnaire}

A novel data collection instrument (questionnaire) to assess knowledge and self-reported practice of HCAIs included 34 questions (with 301 possible answers) in the form of closed-ended (yes or no), contingency, multiple-choice single correct answer, multiple-choice multiple correct answers, and matrix questions. Sample questionnaire will be provided upon request.

\section{Data analysis}

The results were manually entered into an Excel database (Microsoft Corporation, Redmond, WA, USA). Correct answers were taken from the Centers for Disease Control (CDC) guidelines for isolation precautions 2007 [3]. Answers were analysed and categorized according to the CDC's concept of HCAI and standard and isolation precautions (Table 1). A score of 1 was given to every correct question answered, no marks deducted for wrong answers, and unanswered questions were not scored.

Scores were calculated as follows: Overall score $=$ (no. of correct answers earned $\div$ the total no. of correct answers given) $\mathrm{x}$ 100; Categorical score $=$ (no. of correct answers earned in each category $\div$ total no. of correct answers given in each category) x 100 .

\section{Statistical analysis}

Statistical Package for the Social Sciences (SPSS) ver.13 (IBM, SPSS, Chicago, IL, USA) was used for all analyses. Chi-square method was used for analysis of single choice questions; one-way ANOVA method for comparing the overall scores, multiple-choice questions, and some categorical concepts; Kruskal Wallis method for skewed distribution of categorical concepts; and Pearson correlation analysis to find correlations between the overall scores and other variables such as year of education or internship placement. All statistical tests were two-tailed, and Pvalue $<0.05$ was considered statistically significant.

\section{Results}

Overall and categorical scores and influencing factors

The response rate, after excluding the incomplete questionnaire, was $89.2 \%(272 / 305)$. The mean overall score for all students was $52.54 \pm 0.45$ (mean $\pm \mathrm{SE}$ ) out of 100 (Table 2). The majority of students in each cohort $(78.5 \%$ of $5 / 5,76.3 \%$ of $5 / 7,75 \%$ of $6 / 7$, and $81.5 \%$ of $7 / 7$ ) scored between 40 and 59 , and only two students (one each from the $5 / 5$ and $6 / 7$ cohorts) received the score of 71.2. No significant difference in the overall scores was observed among the cohorts ( $\mathrm{P}$ $=0.258)$. When their answers in concept-based categories were analysed (Table 3), students scored well in the knowledge of hand hygiene $(77.57 \pm 0.77)$ and HCAI source $(63.16 \pm 1.18)$. However, their scores were scored relatively weak in the categories regarding personal protective equipment (57.64 \pm $0.60)$, HCW safety $(45.59 \pm 0.86)$, and isolation precautions $(44.59 \pm 0.55)$. The weakest score was in HCA-pathogen identification $(27.44 \pm 0.81)$, which 
Table 1. Categorized HCAI questions

\begin{tabular}{|c|c|}
\hline Category & Questions (type) \\
\hline Pathogens & - Which infectious agents are commonly implicated in health-care associated infections? (MCM) \\
\hline Source & $\begin{array}{l}\text { Which is the most important source/reservoir of health-care associated infections? (MCS) } \\
\text { Which of the followings are the recognized sources of health-care associated infections? (MCM) } \\
\text { Which of the followings from patients do you assume to be infectious? (MCM) } \\
\text { How often do you clean your stethoscope with antiseptic (e.g., } 70 \% \text { alcohol)? (MCS) } \\
\text { With regard to white coats, check the correct answers. (MCM) } \\
\text { How often do you wash your white coat? (MCS) }\end{array}$ \\
\hline $\begin{array}{l}\text { Isolation } \\
\text { precautions }\end{array}$ & $\begin{array}{l}\text { Which of the following behavior(s) can spread infectious organisms? }(\mathrm{MCM}) \\
\text { One is at a very high risk of getting infected via the following actions/behaviors with patients who are } \\
\text { positive for... (MaX, MCM) } \\
\text { How long should be the isolation (cohorting) period for patients with the following diseases? (MaX, } \\
\text { MCM) } \\
\text { When can a patient with pulmonary tuberculosis be removed from the isolation room? (MCS) } \\
\text { With regard to visitor management in hospital settings, which of the following is/are correct? (MCM) } \\
\text { The proper minimum spacing between beds in multi-patient rooms should be ... (MCM) }\end{array}$ \\
\hline Hand Hygiene & $\begin{array}{l}\text { Which is the single most effective method to prevent health-care associated infections? (MCS) } \\
\text { What is the preferred hand washing method to prevent transmission of Clostridium difficile-associated } \\
\text { infections? (MCS) } \\
\text { Do you know how to wash your hands in the six-step hand-washing method? (Y/N) } \\
\text { How do you usually wash your hands while on duty? (MCS) } \\
\text {. When do you wash your hands? (MCM) }\end{array}$ \\
\hline PPE & $\begin{array}{l}\text { Which of the following(s) can effectively prevent spreading or protect you from influenza (flu) during the } \\
\text { flu season? (MCM) } \\
\text {. When do you wear medical utility (non-sterile) gloves? (MCM) } \\
\text { What type of personal protection equipment (PPE) would YOU wear? (MaX, MCM) }\end{array}$ \\
\hline HCW Safety & $\begin{array}{l}\text { Do you assume all unsterile needles and sharps are contaminated? }(\mathrm{Y} / \mathrm{N}) \\
\text { Have you ever been splashed by patient's blood, vomit, or other bodily fluids? (Y/N) } \\
\text { Have you ever sustained a used needle-stick injury? (Y/N) } \\
\text { What action would you take immediately after exposure to HBV- or HIV-contaminated bodily fluid? } \\
\text { (MaX, MCM) } \\
\text {. Where would you dispose of infectious waste from patients? (MCS) }\end{array}$ \\
\hline
\end{tabular}

$\mathrm{Y} / \mathrm{N}$, Yes or No; MCS, multiple-choice single correct answer; MCM, multiple-choice multiple correct answers; MaX, matrix

compromised their mean overall scores. Again, there was no significant difference in the categorical scores among the cohorts. Accordingly, their answers under the knowledge or practice sections also showed no significant difference among the cohorts (data not shown).

Pearson correlation analysis showed no significant correlation between the overall score and the year of education $(\mathrm{r}=0.089, \mathrm{P}=0.144, \mathrm{n}=272)$, or the internship placement $(\mathrm{r}=0.177, \mathrm{P}=0.206, \mathrm{n}=272)$.

\section{HCAI pathogens}

Although a high proportion of students (93.8\%) were aware of the occurrence of HCAIs in their respective hospitals, they could not identify most pathogens commonly implicated. Only $7.7 \%, 16.2 \%$, and $16.9 \%$ of students were able to identify methicillin-resistant Staphylococcus aureus (MRSA), Clostridium difficile, and Mycobacterium tuberculosis, respectively, as HCA pathogens. Importantly, most students were unaware of most viral pathogens that can be acquired during hospitalization, such as rotavirus $(87.0 \%)$, norovirus (Norwalk virus) $(99.6 \%)$, 
Table 2. Number (\%) of medical students with their overall scores in HCAI

\begin{tabular}{|c|c|c|c|c|c|}
\hline & 5-yr program & & 7-yr program & & \\
\hline $\begin{array}{l}\text { Overall } \\
\text { score* }\end{array}$ & $\operatorname{Yr} 5(n=51)$ & $\operatorname{Yr} 5(n=76)$ & Yr $6(n=80)$ & $\operatorname{Yr} 7(n=65)$ & $\begin{array}{c}\text { Total } \\
(\mathrm{n}=\mathbf{2 7 2}) \\
\end{array}$ \\
\hline $75-100$ & $0(0.00)$ & $0(0.00)$ & $0(0.00)$ & $0(0.00)$ & $0(0.00)$ \\
\hline $70-74$ & $1(1.96)$ & $0(0.00)$ & $1(1.25)$ & $0(0.00)$ & $2(0.74)$ \\
\hline $60-69$ & $9(17.64)$ & $10(13.16)$ & $18(22.50)$ & $10(15.38)$ & $47(17.28)$ \\
\hline $50-59$ & $20(39.22)$ & $35(46.05)$ & $36(45.00)$ & $36(55.38)$ & 127 (46.69) \\
\hline $40-49$ & $20(39.26)$ & $23(30.26)$ & $24(30.00)$ & $17(26.15)$ & $84(30.88)$ \\
\hline $30-39$ & $1(1.96)$ & $7(9.21)$ & $1(1.25)$ & $2(3.08)$ & $11(4.04)$ \\
\hline $20-29$ & $0(0.00)$ & $1(1.32)$ & $0(0.00)$ & $0(0.00)$ & $1(0.37)$ \\
\hline $0-19$ & $0(0.00)$ & $0(0.00)$ & $0(0.00)$ & $0(0.00)$ & $0(0.00)$ \\
\hline Mean \pm SE & $52.26 \pm 1.11$ & $51.24 \pm 0.92$ & $53.21 \pm 0.83$ & $53.46 \pm 0.76$ & $52.54 \pm 0.45$ \\
\hline
\end{tabular}

*(no. of correct answers earned/no. of total correct answers given) x 100

respiratory syncytial virus $(84.9 \%)$, and influenza virus $(79.0 \%)$. Of note, only $0.4 \%$ of students knew of norovirus.

\section{Sources of HCAI}

Regarding the recognized sources of HCAIs, $80.9 \%$ of the students correctly identified inpatients as the most important source or reservoir of HCAIs. On the contrary, $48.9 \%$ of students did not know that mucous membranes (such as oral cavity and eyes) of patients could be contagious, and most of them did not consider nasal discharge $(65.08 \%)$, urine $(44.9 \%)$, and vaginal secretions $(72.8 \%)$ from patients potentially infectious.

Despite clear understanding of white coats as the recognized source of infections by $80.9 \%$ of students, $33.5 \%$ of students still thought white coats could prevent HCAI and $43.8 \%$ even considered long-sleeve coats would be more preventative. Furthermore, infrequent washing of white coats was identified in this study: $15.4 \%, 60.7 \%$, and $17.7 \%$ of students, respectively, washed their white coats monthly, weekly, and twice weekly, whereas only $2.2 \%$ of students washed their white coats daily. We also found that only $17.3 \%$ of students in this study cleaned their stethoscopes with antiseptics after examining each patient, $17.7 \%$ daily, $18.8 \%$ weekly, $17.7 \%$ monthly, and $26.5 \%$ never cleaned their stethoscopes. Mercury thermometers are still being used to record axillary temperature in the Chinese hospitals; nevertheless, only $43 \%$ of students recognized them as the source of HCAI. The majority of students (79.8\%) also did not know that environmental surfaces such as bedside curtains, chairs, or cabinets could be infectious sources.

\section{Transmission of HCAI}

We observed a significantly low level of awareness among students concerning the isolation period for various HCAI. For example, less than $10 \%$ of them knew when patients with measles, rubella, mumps, or pertussis should be removed from isolation, and less than $25 \%$ were able to state the correct isolation period of influenza and chicken pox. Interestingly, $21.7 \%$ of students supposed they would remove patients with HIV from isolation only after patients are completely cured. While $46.3 \%$ of students certainly knew the criteria for cessation of isolated patients with pulmonary tuberculosis, the other $53.7 \%$ thought three consecutive negative sputum cultures was required.

When asked about high-risk activities and behaviours, less than $50 \%$ and $30 \%$ of students could correctly identify transmission modes of hepatitis B 
Table 3. Categorical mean scores $\pm \mathrm{SE}^{*}$

\begin{tabular}{|c|c|c|c|c|c|c|}
\hline \multirow[b]{2}{*}{ Category } & \multirow{2}{*}{$\frac{\text { 5-yr program }}{\text { Yr } 5(n=51)}$} & \multicolumn{3}{|c|}{ 7-yr program } & \multirow[t]{2}{*}{$\begin{array}{c}\text { Total } \\
(\mathrm{n}=\mathbf{2 7 2})\end{array}$} & \multirow{2}{*}{$\mathbf{P}$} \\
\hline & & $\operatorname{Yr} 5(n=76)$ & Yr $6(n=80)$ & Yr $7(n=65)$ & & \\
\hline HCA- pathogens ${ }^{b}$ & $26.11 \pm 1.95$ & $27.01 \pm 1.51$ & $27.50 \pm 1.42$ & $28.91 \pm 1.73$ & $27.44 \pm 0.81$ & 0.755 \\
\hline HCAI Source ${ }^{b}$ & $63.26 \pm 2.77$ & $60.32 \pm 2.54$ & $64.54 \pm 2.05$ & $64.70 \pm 2.09$ & $63.16 \pm 1.18$ & 0.713 \\
\hline Isolation precautions $^{a}$ & $46.05 \pm 1.36$ & $43.84 \pm 1.11$ & $44.11 \pm 1.00$ & $44.92 \pm 0.94$ & $44.59 \pm 0.55$ & 0.534 \\
\hline Hand hygiene $^{b}$ & $74.35 \pm 1.86$ & $76.32 \pm 1.49$ & $79.48 \pm 1.37$ & $79.23 \pm 1.52$ & $77.57 \pm 0.77$ & 0.110 \\
\hline $\mathrm{PPE}^{a}$ & $57.44 \pm 1.60$ & $57.07 \pm 0.99$ & $59.17 \pm 1.08$ & $56.60 \pm 1.29$ & $57.64 \pm 0.60$ & 0.399 \\
\hline $\mathrm{HCWS}^{b}$ & $44.12 \pm 2.28$ & $46.71 \pm 1.43$ & $45.00 \pm 1.69$ & $46.15 \pm 1.67$ & $45.59 \pm 0.86$ & 0.738 \\
\hline
\end{tabular}

*(number of correct answers earned in each category/number of total correct answers given in each category) x 100

PPE, personal protective equipment; HCWS, health-care worker safety

$a$ : data in normal distribution, analyzed by One-way ANOVA

$b$ : data in non-normal distribution, analyzed by Kruskal Wallis method

and hepatitis A, respectively. While the majority of students (66\%-94\%) correctly identified viral hepatitis, $\mathrm{HIV}$, and malaria as reportable infectious diseases, only less than $40 \%$ considered influenza and acute viral (haemorrhagic) conjunctivitis as such.

\section{Hand hygiene}

Almost all students (99.6\%) knew the proper handwashing procedure; however, they underestimated its importance because only $52.9 \%$ of them considered it as the most important preventive measure of infection control. We noted encouragingly high levels of selfreported hand hygiene practice before and after examining patients $(96 \%)$, before performing invasive procedures $(92.7 \%)$, and between two different procedures on different patients (89.7\%). However, $58.5 \%$ of students still did not wash their hands between two different procedures on the same patient and $78.3 \%$ before and after touching wounds when they used gloves.

Most students washed their hands with any available means such as water and hand-washing liquid $(60.3 \%)$, water and bar soap (26\%), and water and skin antiseptics (16.2\%). On the other hand, only $23.5 \%$ of students knew hand washing with water and soap is the preferred washing method for prevention of Clostridium difficile-associated infections. Other students thought alcohol hand rub or iodine solution was the right choice.

\section{Personal protective equipment (PPE)}

Excessive or inappropriate use of PPE was noted in this study. For example, caps and shoe covers were routinely required to enter ICU and NICU. In addition, gloves were unnecessarily used by $35.7 \%, 22.4 \%$, and $18.4 \%$ of students for daily routines such as performing physical examination on patients, using patient-care equipment including computers and desks, and making clinical rounds, respectively. Almost all $(95.2 \%)$ students responded that they would (not patients should) wear a mask when transporting coughing patients in and out of wards, whereas $74.3 \%$ would do so when drawing blood from a patient. On the other hand, nearly $80 \%$ of students would not wear a gown and goggles when irrigating a septic wound, nasogastric suctioning, and attending patients with faecal incontinence.

\section{Health-care worker safety}

HCW safety was not well considered by students. Students' knowledge about recommended immunoprophylaxis was variable as $91.9 \%, 41.9 \%$, and $26.8 \%$ of the students respectively considered vaccination of non-immune $\mathrm{HCWs}$ for hepatitis $\mathrm{B}$, chicken pox, and seasonal influenza required. No one answered the post-exposure prophylaxis correctly.

\section{Discussion}

The present study, conducted in response to the discouraging infection control situations at local hospitals, has identified significant limitations in 
students' knowledge and self-reported practices in HCAI.

Despite their relatively low overall scores in the survey, students received high categorical scores in the knowledge and self-reported practice of hand hygiene. Their scores could be attributed to that they learned hand hygiene technique in the preclinical skills training program, and which was reinforced by random assessment during their clinical rotation by the Hospital Infection Control Department and by visual reminders using wall posters near hand-washing sinks. Nevertheless, they lacked good understanding in the hand-washing concepts and thus failed to apply proper hand hygiene knowledge.

One explanation for the students' universal ignorance of important HCA pathogensis that those pathogens are not covered or discussed during the formal lectures and clinical rotation, and that diagnostic tests for them are not available in the participating hospitals. Knowledge deficit about the pathogens, especially those causing reportable diseases, sources, and mode of transmission, could jeopardize the safety of patients and HCWs and outbreak management of nosocomial diseases.

Improper use of PPE among HCWs and students has been reported from different countries. A medical school in the United Kingdom (UK) reported that 35\% of their third-year medical students did not know the correct use of gloves[4], whereas a study in Hong Kong showed only $25.4 \%$ and $44.9 \%$ of nurses used goggles and gowns/aprons, respectively, when they were working with bloody discharge or body fluids [5]. Another nationwide study in Thailand has described the overuse of sterile gloves in $25 \%$ of hospitals [6]. We also noted similar findings in this study. Since no PPE-use guidelines, training, or posters exist in our hospitals, the typical practice of junior students is to follow their seniors, who are also indifferent in this regard. It is apparent from this study and previous reports that while advertently and overly self-protective, HCWs and students are unaware of potential risks.

The World Health Organization (WHO) estimates that about $2.5 \%$ of HIV cases and $40 \%$ of hepatitis B and $\mathrm{C}$ cases among HCWs worldwide are the result of related exposures at work. The self-reported rate $(43.4 \%)$ of needle-stick injury among students in this study is, although lower than that in US (59.4\%) [7] and Germany (58.8\%)[8], still higher than that in a UK teaching hospital $(37 \%)[3,9]$.

According to the CDC, the risk of HIV transmission by an accidental needle prick is very low, at $0.3 \%$ [10]. Also in the Chinese hospital HCAI guidelines, the estimated risk is $0.3-0.5 \%$ [11]. HIV transmission risk from needle-stick injury was, however, identified as high-risk by $95.6 \%$ of our students, which could result in extreme anxiety in $43 \%$ of them who had sustained an accidental needle-stick injury without good knowledge about HIV status of patients for whom the needles had been used. Such overestimation of perceived risks of post-exposure transmission of HBV, HCV, and HIV has also been reported among Georgian HCWs [12].

Despite considerably high incidence and their appreciation of high risk from needle-stick injury, none of the students in this study correctly knew what to do immediately after exposure to HBV- or HIVcontaminated blood, for example via needle prick or cut with sharp objects. In case of accidents, the action most students $(91.9 \%)$ would take was to wash with water and soap, followed by scrubbing and antiseptics; $40.8 \%$ would even use antiseptics to wash mucous membranes, including eyes. Given that underreporting of needle-stick injuries is common among medical students [7] with lack of knowledge about postexposure management, our students are at risk of hepatitis and HIV.

\section{Learning resources of $\mathrm{HCAI}$}

HCAI is taught in most medical schools around the world, but HCAI prevention or infection control is largely ignored or insufficiently addressed in the curricula of most medical schools. For instance, although the prevalence and transmission of HCAI are taught by almost all UK and Irish medical schools, the importance of HCAI as a quality and safety issue is covered in only $60 \%$ of medical schools [13].

Likewise, in SUMC, health-care associated infections are introduced only briefly to preclinical students in the Infection and Immunity module. There are neither formal lectures nor bedside teaching on ward rounds for clinical students during their observership (in the fourth year) and internship (in the fifth year) or even during their two-week elective rotation in the Infectious Disease Department or the regional CDC. Clinical skill assessment after the 48weekclinical internship does not include HCAI-related issues. Actually, no students we casually interviewed were aware of the existence of the hospital infection control guidelines. Self-learning by reading current scientific articles, which has a positive influence on the knowledge and practice of HCWs [14], is still not very popular among Chinese students, with one of the reasons being the language barrier. Therefore, there 
was virtually no educational resource of HCAI for students. As a result, all students, regardless of the medical curriculum, year of education, clinical experience, or internship placement, exhibited a greatly analogous level of knowledge and thus practice of HCAI.

Furthermore, the students' perception of HCAI observed in this study is only that of self-protection, not patient safety. This phenomenon plus other patient safety issues, commonly found in various medical professions, have therefore been recently addressed by the WHO's World Alliance for Patient Safety with a publication "The WHO Patient Safety Curriculum Guide for Medical Schools" [15]. In response to the shortcomings observed in this study, in SUMC we have already introduced health-care safety (i.e., patient and HCW safety) as a case study followed by a targeted lecture to third-year preclinical medical students and plan to assess the impact of this intervention in their final year.

\section{Study limitations}

The survey questionnaire design was based on the US CDC guidelines, which are different from the national or local hospital guidelines, especially in the area of HCAI prevention and control, to address the local problematic issues. Therefore, some questions could be conceptually difficult for students, resulting in random guessing for possible answers. Since there was no penalty for wrong answers or random guessing, the overall or categorical scores may not reflect the students' actual understanding. Furthermore, their compliance with the standard and isolation precautions was self-reported, not observational. Another limitation is due to unavailability of benchmark scores to determine the level of students' knowledge in this study in comparison with that in other studies.

\section{Conclusion}

This study underscores the considerably limited knowledge and practice of students in HCAI, especially in the areas of transmission-based precautions, HCW safety, PPE, and pathogen identification, due to substantial deficiencies in their learning resources. Inadequate preclinical and clinical teaching/training and lack of proper supervision and monitoring of HCW's adherence to infection control and prevention guidelines in the participating hospitals were identified as accountable. As the structure of the curricula and system of most Chinese medical schools are similar, our findings should serve as a basis for future research in medical education and health care in China.

\section{Recommendations}

To minimize HCAI risks associated with HCWs/students and thus with patients, we recommend medical curricula be reviewed and clinical training improved in the area of health-care safety with emphasis on HCAI control and prevention using the local and international guidelines. Specifically, focused teaching on selected issues, such as reportable epidemiologically important pathogens per the institutional guidelines, basic concepts and applications of standard and isolation precautions, and HCW safety according to the CDC's guidelines, and patient safety as recommended by the WHO [15], should be included in the Infection and Immunity curricular module.

Preclinical teaching with didactic lectures and/or problem-based learning (PBL) on $\mathrm{HCW}$ and patient safety issues and clinical training (bedside teaching during dedicated teaching ward rounds or discussion ward rounds) on HCAI following the institutional guidelines should be promoted in the participating hospitals. Using educational posters and flyers and holding seminars/workshops in hospitals are also approaches known to have positive effects on HCW's attitudes and practice. Additionally, theoretical knowledge acquired during the junior (second and third) years should be revised and reinforced in clinical settings.

More importantly, as the clinical practices of mentors and senior doctors have profound influence on the attitudes and practices of the juniors and healthcare students $[9,16]$, surveillance and sustained monitoring of HCWs, followed by interventional measures, such as training and education, are suggested. A monitoring survey such as this study on practicing HCWs in the participating hospitals should be the starting point.

\section{Acknowledgements}

The authors are grateful to the student participants and staff in the first affiliated hospital of SUMC who facilitated the survey, and student volunteers for taking part in the pilot testing. This study was supported by Shantou University Medical College and the $\mathrm{Li} \mathrm{Ka}$ Shing FoundationUniversity of Oxford Global Health Programme (grant no. B9RSRT0-14). The funders had no role in study design, data collection and analysis, decision to publish, or preparation of the manuscript.

\section{References}


1. Allegranzi B, Bagheri Nejad S, Combescure C, Graafmans W, Attar H, Donaldson L, Pittet D (2011) Burden of endemic health-care-associated infection in developing countries: systematic review and meta-analysis. Lancet 377: 228-241.

2. Wu CJ, Gardner GE, Chang AM (2009) Taiwanese nursing students' knowledge, application and confidence with standard and additional precautions in infection control. J Clin Nurs 18: 1105-1112.

3. Siegel JD RE, Jackson M, Chiarello L, and the Healthcare Infection Control Practices Advisory Committee (2007) Guideline for Isolation Precautions: Preventing Transmission of Infectious Agents in Healthcare Settings 2007.

4. Mann CM and Wood A (2006) How much do medical students know about infection control? J Hosp Infect 64: 366370.

5. Chan R, Molassiotis A, Chan E, Chan V, Ho B, Lai CY, Lam P, Shit F, Yiu I(2002) Nurses' knowledge of and compliance with universal precautions in an acute care hospital. Int J Nurs Stud 39: 157-163.

6. Kunaratanapruk S and Silpapojakul K (1998) Unnecessary hospital infection control practices in Thailand: a survey. J Hosp Infect 40: 55-59.

7. Sharma GK, Gilson MM, Nathan H, Makary MA (2009) Needlestick injuries among medical students: incidence and implications. Acad Med 84: 1815-1821.

8. Wicker S, Nurnberger F, Schulze JB, Rabenau HF (2008) Needlestick injuries among German medical students: time to take a different approach? Med Educ 42: 742-745.

9. Stein AD, Makarawo TP, Ahmad MF (2003) A survey of doctors' and nurses' knowledge, attitudes and compliance with infection control guidelines in Birmingham teaching hospitals. J Hosp Infect 54: 68-73.

10. US Public Health Service (2001) Updated US Public Health Service Guidelines for the Management of Occupational Exposures to HBV, HCV, and HIV and Recommendations for Postexposure Prophylaxis. MMWR Recomm Rep 50(RR11): $1-52$.

11. Ministry of Health of the People's Republic of China (2004) Occupational Exposure Prevention Guideline for Health Care Providers Working in HIV/AIDS Care. Available at http://www.moh.gov.cn/mohyzs/pzcxx/200804/32052.shtml Accessed 8 October 2011.

12. Butsashvili M, Kamkamidze G, Umikashvili L, Gvinjilia L, Kankadze K, Berdzuli N (2010) Knowledge of health careassociated infections among Georgian obstetricians and gynecologists. J Infect Dev Ctries 4: 329-333.

13. O'Brien D, Richards J, Walton KE, Phillips MG, Humphreys $H$ (2009) Survey of teaching/learning of healthcare-associated infections in UK and Irish medical schools. J Hosp Infect 73: 171-175.

14. Parmeggiani C, Abbate R, Marinelli P, Angelillo IF (2010) Healthcare workers and health care-associated infections: knowledge, attitudes, and behavior in emergency departments in Italy. BMC Infect Dis 10: 35.

15. Walton $M$, Woodward $H$, Van Staalduinen S, Lemer C, Greaves F, Noble D, Ellis B, Donaldson L, Barraclough B; Expert Group convened by the World Alliance of Patient Safety, as Expert Lead for the Sub-Programme(2010) The WHO patient safety curriculum guide for medical schools. Qual Saf Health Care 19: 542-546.

16. Tavolacci MP, Ladner J, Bailly L, Merle V, Pitrou I, Czernichow P (2008) Prevention of nosocomial infection and standard precautions: knowledge and source of information among healthcare students. Infect Control Hosp Epidemiol 29: 642-647.

\section{Corresponding author}

William Ba-Thein

Department of Microbiology/Immunology and Shantou-Oxford Clinical Research Unit

Shantou University Medical College

Shantou, Guangdong, 515041

P.R. China

Telephone/Fax: +86- (0)-754-88900233

Email: wbathein@stu.edu.cn; williambathein@alumni.mayo.edu

Conflict of interests: No conflict of interests is declared. 